\title{
A INOVAÇÃO NUMA EMPRESA DE BASE TECNOLÓGICA: O CASO DA NEXXERA.
}

\author{
Fernando A. Ribeiro Serra, Manuel Portugal Ferreira, \\ Maricelia de Moraes, Gabriela Fiates*
}

\begin{abstract}
Resumo
O presente trabalho, constituído de um estudo de caso desenvolvido junto à Nexxera Tecnologia e Serviços S.A teve como objetivo avaliar se esta empresa de base tecnológica (EBT) possuía capacidade de inovação que pudesse lhe proporcionar a possibilidade de vantagem competitiva sustentável.A partir do referencial teórico sobre EBTs, inovação e visão baseada em recursos (RBV), foi desenvolvida análise da empresa. Os dados para análise foram coletados por meio de pesquisa bibliográfica, documental e de entrevistas com os sócios e o responsável pelo processo de inovação da empresa. Como resultado, a partir do estudo de caso foi apresentado o caminho utilizado na avaliação da EBT.
\end{abstract}

Palavras chave: Inovação, EBT, estratégia, RBV.

\begin{abstract}
This work presents the case study of Nexxera Tecnologia e Serviços S.A. Its purpose is to evaluate if this technology-based company $(\mathrm{TBC})$ shows innovation capability regarding a sustainable competitive advantage. Departing from the accepted theory of TBC, innovation and resource-based view (RBV), the company analysis was developed. The data and information were collected from bibliographic and documental research, as by personal interviews with the company owners and managers. A way for the analysis is presented and should be tested for other TBC.
\end{abstract}

Keywords: Innovation, TBC, strategy, RBV.

\footnotetext{
* - Fernando A. Ribeiro Serra. Unisul Business School. Universidade do Sul de Santa Catarina. Rua Trajano, 219. Centro Florianópolis - SC. tel: 48-32291914. fernando.serra@unisul.br

- Manuel Portugal Ferreira. Escola Superior de Tecnologia e Gestão. Instituto Politécnico de Leiria. Morro do Lena - Alto Vieiro. Leiria - Portugal. tel:48-32291914. portugal@estg.ipleiria.pt

- Maricelia de Moraes. Universidade do Sul de Santa Catarina. Rua Trajano, 219. Centro Florianópolis - SC. tel: 48-3229I9I4. maricelia.moraes@unisul.br

- Gabriela Fiates. Unisul Business School. Universidade do Sul de Santa Catarina. Rua Trajano, 219. Centro Florianópolis - SC. tel: 48-

32291914. gabriela.fiates@unisul.br
} 


\section{Introdução}

O trabalho clássico de Schumpeter (1934) que analisou a inovação nas firmas sugeria que as pequenas e médias empresas (PME) deveriam ser o veículo mais comum para os avanços tecnológicos e conseqüentemente para o desenvolvimento econômico. Já em trabalhos posteriores, Schumpeter (1942) admitia que as grandes empresas, mesmo em menor número dominavam o mercado, detinham maiores recursos financeiros, físicos e humanos que poderiam dedicar aos esforços de pesquisa e desenvolvimento (P\&D) e inovação - ou seja, detinham vantagens de escala nos processos de (P\&D) - pelo que seriam mais prováveis geradoras de inovações. $O$ que há de comum em ambas as argumentações de Schumpeter (1934, 1942), é que tanto nas PME como nas grandes organizações a inovação é um instrumento vital à sustentação e ao crescimento.

Seguindo a linha de pensamento de Schumpeter (1934, 1942), outros autores como Cyert e March (1963), Tidd, Bessant e Pavit (200I) Freeman (1994) e Dosi (1990) consideram a inovação como fator essencial para a competitividade de firmas e nações. Grant (1996) e Teece $(1998,2000)$ também abordam a importância do conhecimento no processo de inovação e, logo, a importância de as empresas focarem no desenvolvimento interno de conhecimento que possa alimentar resultados inovadores - quer sejam inovações de produto ou processo.

Conseqüentemente, se a inovação é importante para a sustentabilidade de toda e qualquer empresa independentemente de seu tamanho ou ramo de negócios pode-se inferir que para as empresas de base tecnológica isto não é diferente. $\mathrm{Na}$ realidade as EBTs tendem a ser baseadas na incorporação de elevado conhecimento aplicado à tecnologia e, possivelmente a gerar um maior volume de inovações.As EBT assumem cada vez mais importância dentre as PME. Foi constatado, por exemplo, que $55 \%$ das incubadoras de negócios brasileiras são de base tecnológica. (ANPROTEC, 2003).

Apesar da extensa investigação sobre a importância da inovação e do conhecimento a literatura existente apresenta, ainda, diversas lacunas relacionadas aos estudos. Numa perspectiva mais prática falta, por exemplo, a indicação de um caminho que possa ser seguido pelas EBT para a identificação da sua capacidade de inovação.

Este trabalho está organizado em cinco partes.A próxima parte apresenta o referencial teórico que subsidia a pesquisa. $A$ terceira parte descreve o método da pesquisa e é seguida pela apresentação e análise dos dados coletados. Na quinta parte apresentamos uma análise para a identificação da capacidade de inovação das EBT.

\section{Desenvolvimento Conceitual}

Uma premissa basilar do que são EBTs, como veremos à frente, é a sua forte concentração em esforços de P\&D.

Há muitas definições possíveis do que é uma empresa de base tecnologica (PINHO, 2006), mas neste trabalho adotamos a definição de Machado et al., (200I), como:

[...] empresas de base tecnológica são empresas industriais [...], que estão comprometidas com o projeto, desenvolvimento e produção de novos produtos elou processos, caracterizando-se, ainda, pela aplicação sistemática de conhecimento técnico-científico. Estas empresas usam tecnologias inovadoras, têm uma alta proporção de gastos com P\&D, empregam uma alta proporção de pessoal técnico-científico e de engenharia e servem a mercados [...] específicos. (MACHADO et al., 200I, p. 7)

Desta conceituação do que são EBTs podemos sugerir que as EBT, qualquer que seja o mercado a que se dedicam, são empresas que trabalham com inovação e com atividades sistemáticas de P\&D. O trabalho de Torkomian (1992, apud Machado et al. 200 I) salienta algumas características estereotipadas das EBTs que são apresentadas no Quadro I.

Estabelecido que é em que consiste uma EBT, importa entender o que são inovações. Na realidade, há várias definiçõos do que é uma inovação na literatura existente.A importância da inovação tem sido focada por pesquisadores em disciplinas tão diversas como a economia, estratégia e engenharia de produção. $\mathrm{Q}$ Quadro 2 sumaria algumas destas definições.

Embora exista alguma diversidade nas conceptualizações apresentadas, há, também, vários aspectos em comum. No cerne da inovação está a sua comercialização. Ou seja, a inovação está relacionada com a adoção de uma novidade pelo mercado, estritamente ligado ao significado etimológico de 'renovação'. Fica claro também pelas definições que a idéia e as invenções precedem a inovação e que a efetiva realização da inovação requer a sua adoção pelo mercado. Um elemento no esforços contemporâneos de inovação é o esforço conjunto inter-empresas. Quinn (1986), por exemplo, focou como são importantes a interação e os relacionamentos entre organizações enquanto estas partilham a busca de fontes de conhecimento.

Por fim, as inovações podem ocorrer tanto em produtos e serviços, como em processos ou em mercados. Isto é, a entrada em novos mercados de negócio, tecnológicos e geográficos podem ser considerados inovações das empresas.

Embora exista alguma diversidade nas conceptualizações apresentadas, há, também, vários aspectos em comum. No cerne da 


\begin{tabular}{|c|c|}
\hline Característica da EBT & Resultados \\
\hline Motivação para a abertura & $\begin{array}{l}60 \% \text { - identificação de um nicho mercadológico } \\
23,3 \% \text { - desejam de ter um negócio próprio } \\
16,7 \% \text { - possibilidade de aumento dos ganhos }\end{array}$ \\
\hline $\begin{array}{l}\text { Origem da tecnologia } \\
\text { lexperiência }\end{array}$ & $\begin{array}{l}46,7 \% \text { - utilizaram tecnologias desenvolvidas em universidades locais } \\
26,7 \% \text { - utilizou tecnologias desenvolvidas em "fundo de quintal" }\end{array}$ \\
\hline Perfil da empresa & $\begin{array}{l}\text { Setor: } \\
-26,7 \% \text { - novos materiais } \\
\text { - } 16,7 \% \text { - equipamentos industriais } \\
\text { - } 13,3 \% \text { - informática } \\
-13,3 \% \text { - instrumentação } \\
\text { - } 10 \% \text { - automação } \\
\text { Idade: } \\
-63,3 \% \text { - } 0 \text { a } 5 \text { anos } \\
-23,3 \% \text { - } 6 \text { a } 10 \\
\text { Porte: } \\
-63,3 \% \text { - } 0 \text { a } 10 \text { funcionários } \\
-16,7 \% \text { - } 11 \text { a } 20 \\
-13,3 \% \text { - } 21 \text { a } 30\end{array}$ \\
\hline
\end{tabular}

Quadro I - Características das EBT. Fonte: Machado et al., (200I)

\begin{tabular}{|c|c|}
\hline Schumpeter (1934) & $\begin{array}{l}\text { Consiste na introdução de um novo produto no mercado significativamente diferente dos já } \\
\text { existentes, implica uma nova técnica de produção e a abertura de um novo mercado. }\end{array}$ \\
\hline Utterback (197I, p.77) & $\begin{array}{l}\text { "(...) uma invenção que atingiu a fase de introdução no mercado no caso de um novo pro- } \\
\text { duto ou para novo processo, a fase do primeiro uso". }\end{array}$ \\
\hline Quinn (1986, p. 170) & $\begin{array}{l}\text { "um processo interativo e tumultuoso [...] que liga uma rede mundial de fontes de saber às } \\
\text { necessidades sutilmente imprevisíveis dos clientes". }\end{array}$ \\
\hline $\begin{array}{l}\text { Comunidade Européia (CE, } \\
\text { 1996) }\end{array}$ & Consiste na produção, assimilação e exploração bem sucedida da novidade. \\
\hline $\begin{array}{l}\text { Livro Verde da Inovação (CE, } \\
\text { 1996) }\end{array}$ & $\begin{array}{l}\text { Uma renovação e alargamento da gama de produtos e serviços e dos mercados associados; a } \\
\text { criação de novos métodos de produção, de aprovisionamento e de distribuição; a introdução } \\
\text { de alterações na gestão, na organização do trabalho, bem como nas qualificações dos trabal- } \\
\text { hadores. }\end{array}$ \\
\hline Tálamo (2002) & E a disponibilização de uma invenção ao consumo em larga escala. \\
\hline
\end{tabular}

Quadro 2. Definições de Inovação. Fonte: Elaboração dos autores 
inovação está a sua comercialização. Ou seja, a inovação está relacionada com a adoção de uma novidade pelo mercado, estritamente ligado ao significado etimológico de 'renovação'. Fica claro também pelas definições que a idéia e as invenções precedem a inovação e que a efetiva realização da inovação requer a sua adoção pelo mercado. Um elemento no esforços contemporâneos de inovação é o esforço conjunto inter-empresas. Quinn (1986), por exemplo, focou como são importantes a interação e os relacionamentos entre organizações enquanto estas partilham a busca de fontes de conhecimento.

Por fim, as inovações podem ocorrer tanto em produtos e serviços, como em processos ou em mercados. Isto é, a entrada em novos mercados de negócio, tecnológicos e geográficos podem ser considerados inovações das empresas.
A inovação é fundamental à capacidade competitiva das empresas. Diversos autores, como Schumpeter (1934); Hall (1987); Dosi (1990); Chaney et al.(199I); Freeman (1994); Carlsson (1994); Rothwell (1994); Motohashi (1998); Besanko et al. (2000); Klomp e Van Leeuwen (200I); Tidd (200I) focaram o impacto da inovação sobre o desenvolvimento econômico e, em particular, sobre o desempenho empresarial. No entanto, quer os pesquisadores quer os executivos mantém algumas dúvidas sobre quais os fatores ou mecanismos para que a empresa consiga uma vantagem pelas suas inovações. Tidd et al. (1997) apresentam (ver Quadro 3), diversos mecanismos que podem apoiar as empresas na obtenção de vantagens estratégicas pela inovação.

\begin{tabular}{|l|l|l|}
\hline \multicolumn{1}{|c|}{ MECANISMO } & \multicolumn{1}{|c|}{ VANTAGEM ESTRATEGICA } & \multicolumn{1}{c|}{ EXEMPLOS } \\
\hline $\begin{array}{l}\text { Novidade no produto ou ser- } \\
\text { viço }\end{array}$ & Oferecer algo que ninguém foi capaz. & Walkman, máquina de lavar pratos... \\
\hline Novidade no processo & Mais rápido, menos custo, mais personalizado. & Banco pela Internet, vendas on-line... \\
\hline Complexidade & $\begin{array}{l}\text { Disponibilizar o que os outros têm dificulda- } \\
\text { des em dominar }\end{array}$ & A Rolls-Royce, os motores de avião... \\
\hline $\begin{array}{l}\text { Proteção legal á propriedade } \\
\text { intelectual }\end{array}$ & Pagar pela licença ou quota & Os Medicamentos (Zantac, Prozac). \\
\hline $\begin{array}{l}\text { Fatores competitivos: } \\
\text { adição/extensão }\end{array}$ & Qualidade, preço. & A produção automóvel japonesa \\
\hline Timing & $\begin{array}{l}\text {-Vantagem de ser o primeiro a avançar; } \\
\text {-Vantagem dos rápidos seguidores. }\end{array}$ & $\begin{array}{l}\text { - Amazon.com e o Yahoo; } \\
\text { - Palm Pilot e outros computadores de bolso }\end{array}$ \\
\hline Projeto consistente & $\begin{array}{l}\text { Base de outras variantes e gerações que pos- } \\
\text { sam sustentar }\end{array}$ & O Boeing 737 com mais de 30 anos \\
\hline Redefinição das regras & $\begin{array}{l}\text { Criar qualquer coisa, conceito novo, que façam } \\
\text { anteriores serem obsoletas. }\end{array}$ & $\begin{array}{l}\text { Máquinas de escrever x processadores de } \\
\text { texto do computador, gelo x frigorífico, lâm- } \\
\text { pada elétrica x candeeiros a gás ou a petró- } \\
\text { leo. }\end{array}$ \\
\hline Reconfiguração das partes & $\begin{array}{l}\text { Construir redes mais eficazes, subcontratar e e } \\
\text { coordenar uma empresa virtual. }\end{array}$ & $\begin{array}{l}\text { A Benetton nos tecidos, a Dell nos computa- } \\
\text { dores. }\end{array}$ \\
\hline
\end{tabular}

Quadro 3 -Vantagens estratégicas pela inovação. Fonte:Tidd et al. (1997)

A análise do Quadro 3, acima, permite inferir que para obter vantagens estratégicas pelas inovações as organizações podem se utilizar de diferentes mecanismos. De fato, algumas das empresas de maior sucesso conseguiram desenvolver inovações que se verificou terem maior aceitação pelo mercado.

\section{O Tipo de Inovações Gerado pela EBTs}

Damanpour (199I) identificou uma inovação organizacional como um equipamento, sistema, política, processo, produto ou serviço que é gerado internamente ou adquirido externamente e novo para a organização que o adota.Assim, a inovação pode representar uma forma de transformação de uma organização, seja pela resposta às mudanças em seu ambiente interno ou externo, ou seja, pela decisão consciente e voluntária de influenciar seu ambiente.

Para que a inovação ocorra é preciso gerenciar o processo de inovação, pela avaliação do ambiente interno e externo, pela escolha das inovações potenciais a serem desenvolvidas, pela alo- 
cação dos recursos para seu desenvolvimento, e pela implementação (e aprendizado), fundamentais para ser uma "empresa inovadora" (TIDD et al., 1997).

Há diversos tipos de inovações concetualizados na literatura existente na área de estratégia. Daft (1978); Kimberly e Evanisko (I98I); e Damanpour (1987) classificaram as inovações em administrativas e técnicas. Possivelmente a classificação mais reconhecida é a que distingue entre inovações incrementais e inovações radicais (ver ETTLIE; BRIDGES; O`KEEFE, 1984; NORD; TUCKER, 1987). Também a tipologia de Henderson e Clark (1990) distinguindo entre inovação arquitetural e de componente faz a distinção entre os tipos de inovação.

Tidd et al., (200I) analisam as inovações segundo dois aspectos: pelo objeto da mudança, ou seja, o que é mudado, e pela percepção da mudança pelo mercado, ou seja, a extensão percebida da mudança. Tidd et al., (200 I), propõem que em função "do que é mudado", a inovação seja de produto, serviço ou processo. Por outro lado, considerando a extensão percebida da mudança, a inovação pode ser classificada como: incremental, radical ou distintiva, e de transformação ou revolucionária, como descrevemos no Quadro 4.

\begin{tabular}{|c|l|l|}
\hline Inovação Incremental & $\begin{array}{l}\text { Na inovação incremental, o novo produto, serviço ou processo mantendo as suas funções básicas, } \\
\text { incorpora novos elementos em relação ao anterior (BAPTISTA, I999), ou seja, objetiva-se melhorar } \\
\text { o desempenho e a funcionalidade dos produtos, serviços e processos para atender a determinados } \\
\text { consumidores ou para reduzir os custos, por exemplo. (LYNN;AKGÜN, I998). }\end{array}$ \\
\hline Inovação Radical ou \\
Distintiva & $\begin{array}{l}\text { Na inovação radical ou distintiva, apesar do produto, serviço ou processo manter as características } \\
\text { daquele a partir do qual foi desenvolvido, apresentará novas características que proporcionam fun- } \\
\text { ções que não existiam no original (BAPTISTA, I999). Considerando a classificação de Lynn e Akgün } \\
\text { (I998), neste tipo de inovações podem acontecer duas situações em relação às incertezas de mer- } \\
\text { cado. As evolucionárias de tecnologia, que buscam desenvolver produtos ainda não conhecidos } \\
\text { para necessidades já bem conhecidas do mercado. Segundo os autores acontece quando uma orga- } \\
\text { nização não domina determinada tecnologia ou não conhece formas para diminuírem os seus cus- } \\
\text { tos de desenvolvimento e produção para serem competitivos no mercado. As evolucionárias de } \\
\text { mercado buscam implementar tecnologias existentes em um mercado novo e desconhecido. } \\
\text { Existe uma grande incerteza dos mercados em relação à aceitação dos produtos. }\end{array}$ \\
$\begin{array}{c}\text { Inovação de } \\
\text { Transformação ou } \\
\text { Revolucionária }\end{array}$ & $\begin{array}{l}\text { Na inovação de transformação ou revolucionária, novos produtos, serviços ou processos aparecem } \\
\text { para satisfazer uma necessidade ou criar uma nova necessidade, sem qualquer relação com o que } \\
\text { existia antes. Estas inovações são mais complexas e com altos níveis de incertezas, tanto de mer- } \\
\text { cado como técnicas. Apesar do custo e risco envolvidos podem proporcionar uma vantagem com- } \\
\text { petitiva mais sustentável para as organizações que implementarem-na. }\end{array}$ \\
\hline
\end{tabular}

Quadro 4: Classificação da inovação segundo aspecto percepção da mudança

Fonte: Elaboração dos autores

\section{O Papel dos Recursos para o Sucesso da EBT}

Ao empreender esforços de inovação as empresas não apenas alavancam os seus recursos para novos usos como também constroem novos recursos estratégicos, competências ou capacidades estratégicas. A teoria Baseada em Recursos (RBV), baseada nos trabalhos de autores como Penrose (I959), Rumelt (1984), Wernerfelt (1984); Barney (1991) e Peteraf (1993), entre outros, sustenta que o crescimento e sucesso das organizações estão ligados ao controle e uso efetivo de recursos heterogêneos e únicos. Para gerarem vantagem competitiva os recursos precisam ser valiosos e raros, difíceis de serem imitados e substituídos - estes recursos são comumente designados pela sigla "VRIN".
O Quadro 5 apresenta a classificação proposto por Jay Barney (1991) do que constituem recursos estratégicos. A empresa que detem recursos estratégicos com estas características pode alcançar uma vantagem competitiva. Na medida que estes recursos não sejam imitáveis ou substituíveis, permitem à empresa alcançar uma vantagem competitiva sustentável no tempo.

Para Wernerfelt (1984), os recursos das organizações podem ser tangíveis e intangíveis. Barney (1991), contribuindo para a classificação dos recursos os apresenta como: recursos de capital físico - como tecnologia física (equipamentos), acesso a insumos etc.; recursos de capital humano - como treinamento, experiência, inteligência, relacionamentos e capacidade gerencial 


\begin{tabular}{|l|l|}
\hline Recursos VRIN & \multicolumn{1}{|c|}{ Descrição } \\
\hline Valiosos & $\begin{array}{l}\text { Recursos que permitam à organização formular estratégias que melhorem sua eficiência e eficácia, apro- } \\
\text { veitando oportunidades ou minimizando as ameaças. }\end{array}$ \\
\hline Raros & $\begin{array}{l}\text { Recursos valiosos não pode ser fonte de vantagem competitiva sustentável se são acessíveis a vários } \\
\text { competidores, uma vez que cada um de seus detentores terá a capacidade de explorá-los, implementando } \\
\text { estratégias comuns, o que levaria a obterem apenas retornos médios. }\end{array}$ \\
\hline $\begin{array}{l}\text { Imperfeitamente } \\
\text { imitáveis }\end{array}$ & $\begin{array}{l}\text { Mesmo que sejam valiosos e raros, os recursos só serão fonte de vantagem competitiva sustentável se as } \\
\text { organizações que ainda não os possuem não puderem obtê-los com demasiada facilidade. }\end{array}$ \\
\hline Não substituíveis & $\begin{array}{l}\text { Os recursos devem ser não-substituíveis, ou seja, não devem possuir um equivalente que não seja raro e } \\
\text { imperfeitamente imitável. Pois a imitação, ou a substituição por outro recurso estratégico é uma ameaça à } \\
\text { sustentabilidade da vantagem estratégica, tornando o recurso original obsoleto, perdendo sua capacidade } \\
\text { de gerar valor. }\end{array}$ \\
\hline
\end{tabular}

Quadro 5 - Recursos Estratégicos

Fonte: Barney (I99I); Dierickx; Cool (I989); Reed; DeFillipi (1990).

individual; e recursos de capital organizacional - como a estrutura formal da organização, o planejamento formal e informal e sistemas de coordenação e controle, bem como relacionamentos informais, cultura organizacional, etc.

Os recursos intangíveis, como por exemplo, capital humano, segundo Dierickx e Cool (1989), estão associados à organização e não podem ser adquiridos no mercado dos fatores que Barney (1986) descreve. Pelo menos, não podem ser comprados da mesma forma que o podem ser os recursos tangíveis ou físicos. Os recursos intengíveis precisam ser desenvolvidos, desdobrados e, acumulados, o que exige tempo e relações entre recursos (DIERICKX e COOL, 1989). Desta forma, por serem mais raros e mais difíceis de imitar, poderão ser a fonte mais provável de uma vantagem competitiva sustentável. (MICHALISIN, SMITH e KLINE, 1997).

Os recursos estratégicos tendem a ser recursos intangíveis por natureza, mas nem todos os recursos intangíveis são estratégicos (MICHALISIN, SMITH e KLINE, 1997). É, assim, razoável sugerir que o conhecimento, base fundamental para a inovação, pode ser considerado como um recurso VRIN, pelo seu valor, raridade, difícil imitação e não ser facilmente substituível. Esta sugestão está de acordo com Grant (1996) e Tallman et al. (2004) que os recursos e as capacidades baseadas no conhecimento (knowledge-based capabilities) são um pilar essencial da vantagem competitiva das organizações.. As inovações baseadas no conhecimento impactam positivamente o desempenho das organizações. (MCGRATH; MACMILLAN, 2000).

\section{Metodologia}

Em função da natureza do problema a abordagem do estudo de um caso é qualitativa. Quanto aos fins, foi definida como descritiva, porque pretende apresentar de forma mais ou menos detalhada a realidade investigada (VERGARA, 1997) já que tem como objetivo propor um caminho a ser seguido pelas empresas de base tecnológica para identificar sua capacidade de inovação visando à vantagem competitiva. Quanto aos meios, trata-se de uma pesquisa bibliográfica, documental e de estudo de caso. (VERGARA 1997; GODOY, 1995 a, b).

O estudo de caso pode ser considerado como estratégia relevante em certas situações: ao tentar responder as questões: Como? Por quê? (YIN, 2005). Yin (2005) acrescenta que os casos únicos devem ser raros ou reveladores. $O$ caso analisado é ilustrativo das capacidades de uma EBT. Ainda, o estudo de caso foi realizado no estado de Santa Catarina, que se destaca pela concentração de empresas de base tecnológica.

A empresa Nexxera Tecnologia e Serviços S.A foi escolhida para o estudo, uma vez que está ligada ao ramo de informática, é dedicada a um nicho de mercado não usual e compete com empresas estrangeiras de grande porte; o que já a torna rara. O caso da Nexxera foi escolhido também por tratar-se de uma empresa que apresenta características similares às de várias outras empresas da região de Florianópolis/SC, o que pode tornála representativa. Pese o fato de não procurarmos generalizar a partir de um caso, poderemos retirar deste caso inferências eventualmente extensíveis a casos similares.

Os dados primários foram coletados por meio de entrevistas semi-estruturadas com os sócios e com o responsável pela área de desenvolvimento de produtos. Para a pesquisa bibliográfica recorremos a livros e artigos publicados em eventos e revistas científicas, bem como a documentos internos da empresa. Com o recurso a várias fontes de dados respitamos o preceito descrito por Yin (2005) para a coleta de dados. Por outro lado, a utilização de múltiplas fontes de evidência possibilita a triangulação de informação. 


\section{O Caso da Nexxera}

A Nexxera Tecnologia e Serviços S.A. é uma BSP - Business Service Provider- voltada para a troca eletrônica de dados e provedora dos serviços como infra-estrutura para a interconexão de parceiros de negócios, gestão das informações trafegadas e tomada de decisão, soluções para automação da integração dos parceiros, agregando valor às redes de relacionamento e negócios. A Nexxera é especializada em soluções de gerenciamento financeiro - aplicativos de valor agregado para os segmentos: financeiro, industrial, varejo/distribuidor, concessionárias, dentre outros.A Nexxera controla o grupo de empresas responsáveis pelo desenvolvimento de aplicativos de cash management, portais de compra e governança eletrônica e comercialização de soluções B2B, B2G e B2C. Em suma, a Nexxera é uma prestadora de serviços de base tecnológica que enfatiza a integração de informações entre agentes (players).

A Nexxera existe há 15 anos, Foi fundada em 1992, possui mais de 32 mil pontos integrados no Brasil e no exterior e contava em 2007 com 106 funcionários. A Nexxera ocupa hoje a terceira posição no ranking das empresas de TI, especializadas em EDI no Brasil.

A Nexxera identificou a necessidade de realizar investimentos em pesquisa e desenvolvimento. Nos últimos anos, a Nexxera buscou no mercado empresas fortes na concepção e desenvolvimento de ferramentas tecnológicas como a SIG, dataware house, CRM, EDI, realizou alianças, investiu na criação de novas empresas e na participação em organizações estratégicas.Atualmente possui, também, laboratórios próprios para desenvolvi- mento tecnológico.

\section{A Inovação na Estratégia da Nexxera}

Os negócios da Nexxera são compostos por um conjunto de soluções voltadas à infra-estrutura e gerenciamento de logística financeira das empresas. Atualmente, a empresa conta com representação comercial nos principais centros de negócio do país, com o slogan "inovação é o nosso compromisso".

A Nexera nasceu na beira da praia dos Ingleses, 3 pessoas de tecnologia (eu, Rui e Ademir) em 1989. Surgiu o papo, vamos fazer algo diferente, inovador, para que sejamos reconhecidos, já que como funcionários não fomos. (Edson Silva, presidente da Nexxera)

A Nexxera possui uma equipe de desenvolvimento, o que provavelmente reflete a orientação do Top Management, como ilustra a declaração de seu Sócio e Vice-presidente, Rui Muller:"(a Nexxera é) totalmente envolvida por conhecimento tecnológico, é totalmente inovadora”. Ou, segundo o Presidente Edson Silva:

"a empresa ocupa um patamar diferencial [...], é pioneira em inovação...”. Onde, “o conhecimento humano é o maior patrimônio da Nexxera [...], dependemos destas pessoas para criar".

As necessidades do mercado orientaram, pelo menos em parte, os esforços da Nexxera para o desenvolvimento de produtos e serviços. $\bigcirc$ Quadro 6 sintetiza a evolução das inovações introduzidas pela Nexxera, e qual a necessidade de mercado em que foi baseada.

\begin{tabular}{|l|c|c|l|}
\hline \multicolumn{1}{|c|}{ Produto/Serviço } & $\begin{array}{c}\text { Quem } \\
\text { desenvolveu? }\end{array}$ & Qual ano? & \multicolumn{1}{|c|}{ A partir de qual necessidade? } \\
\hline $\begin{array}{l}\text { Ambiente Eletrônico de Ne- } \\
\text { gócios }\end{array}$ & Nexxera & 2007 & $\begin{array}{l}\text { Atendimento à demanda de mercado no âmbito de in- } \\
\text { tegração financeira total das operações financeiras } \\
\text { dos clientes. }\end{array}$ \\
\hline Pagamento Eletrônico & Nexxera & Outubro/2005 & $\begin{array}{l}\text { Demanda de clientes, necessidade do mercado, substi- } \\
\text { tuição de ferramenta existente. Gerenciamento das } \\
\text { contas a pagar do cliente. }\end{array}$ \\
\hline Cobrança Eletrônica & Nexxera & Maio/2005 & $\begin{array}{l}\text { Demanda de clientes, necessidade do mercado, substi- } \\
\text { tuição de ferramenta existente. } \\
\text { Gerenciamento das contas a receber do cliente. }\end{array}$ \\
\hline Varredura Eletrônica & Nexxera & 2006 & $\begin{array}{l}\text { Demanda de clientes, necessidade do mercado, efetuar } \\
\text { rastreamento de todos os documentos a pagar do } \\
\text { cliente. }\end{array}$ \\
\hline ConsigWeb & Nexxera & Maio/2005 & $\begin{array}{l}\text { Demanda inicial do Banco ABN - redução do custo } \\
\text { operacional do processo de autorização de débito do } \\
\text { valor das parcelas dos empréstimos consignados. }\end{array}$ \\
\hline
\end{tabular}


J.Technol. Manag. Innov. 2008, Volume 3, Issue 3

\begin{tabular}{|l|c|c|l|}
\hline NexxCash Bank & $\begin{array}{c}\text { EloSoft } \\
\text { (Fornecedor } \\
\text { Nexxera) }\end{array}$ & 2007 & $\begin{array}{l}\text { Demanda de mercado, consolidação de extratos / con- } \\
\text { tas bancárias. }\end{array}$ \\
\hline Arrecard & $\begin{array}{c}\text { TechPeople } \\
\text { (Nexxera) }\end{array}$ & 2007 & $\begin{array}{l}\text { Demanda de mercado, gerenciamento das arrecada- } \\
\text { ções pelas concessionárias. }\end{array}$ \\
\hline PAG-FLEX & IsNet (parceria) & Março/2005 & $\begin{array}{l}\text { Demanda do Banco do Brasil - necessidade de ofertar } \\
\text { solução que viabilizasse flexibilidade de pagamento } \\
\text { para conquistar cliente Basf. }\end{array}$ \\
\hline SkyLine & Nexxera & 1995 & $\begin{array}{l}\text { Demanda de clientes Tramontina e Massas Filler, aten- } \\
\text { dimento ao mercado de EDI. }\end{array}$ \\
\hline Vida Fácil & Nexxera & 2006 & $\begin{array}{l}\text { Demanda de mercado, pagamento de contas por celu- } \\
\text { lar. }\end{array}$ \\
\hline
\end{tabular}

Quadro 6 - Evolução da Nexxera

As EBT são empresas que trabalham com inovação e com fortes atividades de P\&D, como é o caso da Nexxera. No entanto, os esforços de P\&D são direcionados para as necessidades de um nicho do mercado que é previamente indeitifcado. Esta observação parece suportar que as Nexxera como EBT, se focam num nicho tecnológico e que para o serviço deste nicho é fundamental deter conhecimento tecnológico. $\mathrm{Na}$ realidade, a oportunidade detectada é mais abrangente e resulta da lacuna existente no fornecimento de soluções tecnológicas para comunicação de dados, inicialmente baseada no conhecimento individual de seus empreendedores. Isto fica claro das entrevistas realizadas aos Excutivos.Ainda, a Nexxera, além de possuir uma equipe de desenvolvimento, tem como prática organizacional firmar acordos com parceiros para o desenvolvimento de produtos.

Segundo Rui Muller, vice-presidente da empresa

O projeto “Vida Fácil”, foi desenvolvido para a rede bancária e beneficia a todos os usuários, podendo ser usada de forma útil e prática pela internet, telefone móvel, com serviços de transferências, pagamentos e caracteriza-se como um dos frutos da parceria da NEXXERA com a Universidade do Sul de Santa Catarina - Unisul.

Um trabalho desenvolvido é o "Vida Fácil”, (...) A equipe deste projeto estuda na UNISUL, que funciona como pilotos, que já pagam suas mensalidades via celular. (Oswaldo Malheiros, assessor de Tl)

Considerando que as inovações da empresa, embora sendo principalmente de produto, causam mudanças significativas de processo para os clientes que as utilizam. A percepção do tipo de inovação que a empresa gera é um indicador interessante da postura de inovação e posicionamento de mercado que os li- deres visionam. Esta percepção pode não corresponder à definição estrita que é acadêmicamente estabelecida. Por exemplo, um Executivo pode considerar que a inovação é radical muito embora esta não altere totalmente a forma como o agente usa, utiliza, consume ou se comporta pela sua mera utilizção. No caso da Nexxera os Executivos afirmam as suas inovações como sendo radicais, apesar de manterem as funções características similares às oferecidas pelos concorrentes, todos estrangeiros. A maior parte das inovações foi considerada radical, visto que aconteceram em um ambiente complexo e dinâmico em tecnologia e que buscavam resolver uma necessidade relativamente nova, conforme evidenciado nas entrevistas aos Executivos:

A característica da empresa sempre foi inovação, nós não desenvolvemos tecnologia e soluções para determinadas tendências do mercado e sim fomos atrás do que estava acontecendo para atender, oferecer e dinamizar o mercado com soluções diferenciais competitivos, agregar valor nas soluções. (Edson Silva, presidente da Nexxera)

A NEXXERA é totalmente inovadora, estamos no mercado há 15 anos, não copiamos nada de outro que faz e muito menos fazer igual ou tentar melhorar o que já está feito, esta é uma das características da NEXXERA. (Rui Muller, vice-presidente da Nexxera)

A NEXXERA é inovadora por propor ao mercado uma solução que ainda não demandou.A UNISUL é nossa cliente e a solução oferecida a ela é a logística financeira que o mercado em geral ainda não adota por isso a empresa é inovadora. O mercado não usa toda a sua potencialidade. É uma solução que diminui custo, oferece agilidade, agrega segurança, gera competitividade para as empresas que ainda não se vê na prática, sendo um fato de inovação. (Oswaldo Malheiros, assessor de TI) 
Uma análise das inovações introduzidas pela Nexxera permite concluir que estas apresentam diferenciais que o mercado valoriza, mas não permite concluir que são, efetivamente, radicais. O incrementalismo é notório, ainda, nos contínuos desenvolvimentos e adaptações de produto e processo - o que materializa as inovações incrementais para melhorar a funcionalidade dos produtos existentes.

Pode-se propor a partir do referencial teórico utilizado, que o sucesso das organizações mais inovadoras, como imaginamos a Nexxera, poderia ser aferido pela viabilidade comercial das suas invenções; fossem estas inovações nos produtos, serviços ou processos. Podemos avançar, também, que as inovações geradas dariam origem a um fluxo subsequente de novas inovações baseadas nas invenções originais. $O$ caso da Nexxera mostra uma empresa de sucesso com forte vocação inovadora. Mostra também as constantes adaptações de produtos/serviços e processos que são personalizados às necessidades concretas de cada cliente.

Quais os recursos que a Nexxera dispoe e como estes contribuem para a capacidade inovadora? O referencial teórico nos permite sugerir que a posse de recursos VRIN seria essencial à empresa para esta gerar inovações. Efetivamente, ao longo do estudo identificamos diversos recursos organizacionais importantes na Nexxera. A imagem de empresa inovadora com evidências objetivas pelos prêmios adquiridos:

A premiação é adequada, mas queremos sempre mais, em 2004 e 2005 fomos os primeiros, e em 2006 não competimos para não ficar repetitivo, então não nos inscrevemos. Porque não vale a pena ficar competindo o tempo todo na mesma coisa. $O$ bom mesmo é dar um tempo maior para apresentar novos serviços e produtos que tiveram a melhor absorção no mercado e assim, melhor fidelizar o cliente. (Edson Silva, presidente da Nexxera.)

A capacidade de desenvolvimento de produtos, a partir do seu processo formalizado e potencializado à partir de 1995, pelos seus funcionários e a liderança e equipe executiva da Nexxera são fundamentais:

Incentivando os funcionários e todos os produtos surgirem com diferencial competitivo, vendo as tendências do futuro e não copiando que os outros fazem. Mais a empresa ocupou um patamar diferencial, um espaço importante no mercado Nacional e Internacional. Pioneira inovadora, com custo alto não só em pesquisa e desenvolvimento, mas, principalmente levar para o mercado, e que demora a acontecer. (...) O diferencial da NEXXERA é investir em pesquisa tecnológica, com uma equipe de gestores técnicos, com a missão de converter todo o resultado da pesquisa em produto. (...) Existem outros cursos temporários de 6 meses, de finais de semana, só para não ficar parado, pois a tec- nologia exige muito esforço mental e transformar todo este conhecimento em resultado. A rotatividade é tão baixo que não chega a 5\%. (Edson Silva, presidente da Nexxera)

A capacidade de gestão dos recursos, conhecimento e resultados é outra capacidade desenvolvida na Nexxera:

$O$ reconhecimento do pessoal é financeiro, tendo participação nos lucros da empresa e por competências, isto ocorre quadrimestralmente. A NEXXERA atende ao reajuste salarial, até mais que o mercado oferece, temos o Plano de Cargos e Salários, tudo para a motivação geral. Existe o incentivo aos estudos, onde temos a parceria com a UNISUL. Os gestores não possuem salas individuais, tudo é aberto e junto com a parte operacional, existindo maior integração.A pesquisa de clima organizacional é feito uma vez por ano.

Trabalhamos com ferramenta gerencial de dados SIG, que é importante para os processos da empresa, tudo que acontece dentro e fora da empresa ficam integrados, para não se cometer erros e falhas freqüentes, deu problema deve ser resolvido para que nunca mais ocorra, pois isso gera custos e tempo para empresa. (Rui Muller, vp da Nexxera.)

A capacidade de gestão financeira da Nexxera parece ser importante para a sustentação da estratégia voltada para a inovação. A relação das pessoas com a inovação está ligada à construção de uma cultura para a inovação:

Investir em pessoas, incentivar, mas tudo com recurso próprio, por 3 anos e meio e após os resultados. Obtivemos um equilibrio e ter passos maiores, mas até então tudo com recurso próprio. A estratégia da inovação com a empresa faz parte, está na cultura da empresa, uma obrigação nossa, tem que estar no sangue de cada um. Inovação e empresa é espírito de casados. ( Edson Silva, presidente da Nexxera.)

Temos uma equipe reduzida, umas com experiência maior, outros especialistas, outros com visão estratégica, então fizemos a junção de todos. Sendo então que não podemos deixar de incentivar para não afetar no trabalho.Aprende-se com a inovação constantemente, é um feeling, acompanha a evolução do mercado. (Edson Silva, presidente da Nexxera.)

\section{Discussão}

Este trabalho procurou responder se a Nexxera, uma empresa de base tecnológica de Santa Catarina no Brasil, pode ser considerada uma empresa inovadora à partir da análise com base em referencial teórico calcado em conceitos de inovação e da abordagem estratégica da RBV.A pergunta de pesquisa se justifica, visto que a disputa pelo conhecimento (GRANT, I996; 
TEECE, 1998, 2000) e a inovação (MCGRATH et al, 1996) estão cada vez mais presentes nos textos que tratam da competitividade nas organizações (ver por exemplo, DUNNING, 1995; TEECE, 1998; BARTLETT e GHOSHAL, 1989; TEECE, 2000; MARCH, I99I).

A partir do referencial teórico foi realizada a avaliação da Nexxera, a partir do caminho apresentado na Figura I. Consideramos que as EBT são criadas a partir do aproveitamento de um nicho ligado ao conhecimento que domina em seu nascimento. Para tanto a empresa se aproxima de universidades para desenvolver conhecimento e para identificar de recursos humanos e tecnológicos importantes para a inovação. Outras redes de relacionamento com organizações complementares e mesmo concorrentes são também desenvolvidas.As inovações são eventualmente radicais no início, recebendo inovações incrementais para sustentar o crescimento a partir do atendimento das necessidades e oportunidades do mercado. $O$ desenvolvimento das inovações e dos relacionamentos acontece por um processo. $O$ resultado da inovação se reflete pela aceitação pelo mercado refletida no market share e/ou no crescimento do faturamento. Para sustentar sua vantagem competitiva a EBT depende do acesso a recursos VRIN baseados em conhecimento. Adicionalmente, o trabalho propiciou identificar o modelo de processo de desenvolvimento de inovações, segundo os modelos apresentados por Rothwell (1993) e identificar recursos VRIN da Nexxera.
A EBT indentifica um nicho à partir do conhecimento tecnológico que domina.

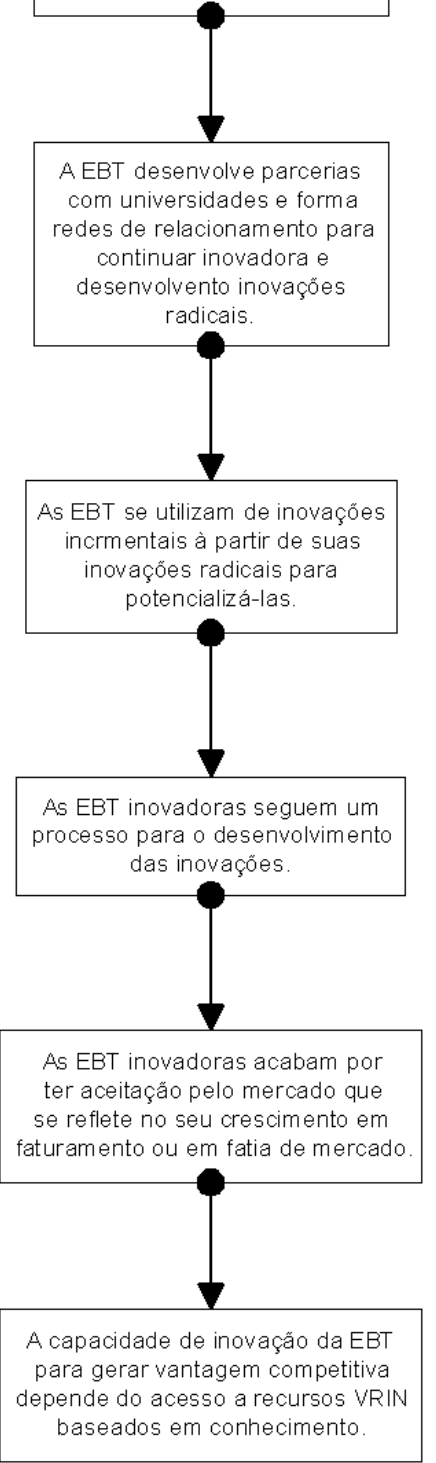

P1 - A maior parte das EBT considera a oportunidade ao se dedicar a um nicho tecnológicc o que leva a propor que o conhecimento tecnológico é fundamental.

P2 - E que este conhecimento tecnológico foi fundamentalmente desenvolvido n universidade, o que leva a propor que o relacionamento com a universidade mesmo não se tratando de uma empresa diretamente ligada a ela deve ser importante.

P3 - O tipo de inovação deve infiuenciar o processo de inovação que é complexo e depende de redes de relacionamento.

P5 - Aparentemente, as organizações inovadoras a partir de inovações radicais ou de transformação vão continuar inovando a partir destas com inovações incrementais.

P10 - A capacidade de inovação depende de um processo formal ou informal de gestão do conhecimento organizacional

P7 - Organizações inovadoras podem ser identificadas pela aceitação de suas 'invenções' como inovação em produtos, serviços ou processo pelo mercado.

P9 - A capacidade de gerar inovaçóes dependerá do acesso a recursos VR/N baseados em. conhecimento.

Figura I - Caminho para avaliação da EBT Nexxera 
As informações acima indicam que o tipo de inovação deve influenciar o processo de inovação que é complexo e depende das redes de relacionamento existentes.

Com relação ao desempenho da empresa e seus resultados financeiros a NEXXERA possui sua tecnologia própria, e a ligação direta com seus clientes, e podendo cobrar $30 \%$ a menos que a concorrência e cobrava somente o uso do software. A concorrência dependia de serviços terceirizados e por isso cobra mais caro e a vantagem da NEXXERA é não possuir serviços terceirizados o que gera um custo bem menor.

Os riscos do mercado são o de não absorver a inovação, sendo que a inovação tem um risco constante. (Edson Silva, presidente da Nexxera.)

Tem que perceber o que tem no produto/serviço, sem isso não tem utilidade alguma. Então, tem que gastar com marketing, mídia para explicar ao consumidor qual o beneficio do produto/serviço. Logo a inovação é perigosa. Existe a inovação recorrente na qual se agrega valor, dar uma função a mais. Sendo que este tipo de inovação não contém risco algum, pois o cliente já conhece a base principal. (...) E o nosso trabalho não é agregar valor e sim colocar algo novo no mercado, por isso o risco da inovação perigosa. (Rui Muller, vice-presidente da Nexxera)

O caminho para avaliação apresentado na Figura I pode ser talvez adequado para identificação de empresas de base tecnológica as proposições ordenadas possibilitaram um caminho sugerido para as EBT inovadoras. Este caminho propõe que as EBT são criadas a partir do aproveitamento de um nicho ligado ao conhecimento que domina em seu nascimento. A partir de então solidifica ou estabelece uma proximidade com universidades para o desenvolvimento de conhecimento e para a identificação de recursos humanos e tecnológicos necessários para o desenvolvimento de inovações. Outras redes de relacionamento com organizações complementares e mesmo concorrentes são desenvolvidas para sustentar o crescimento pelas inovações. As inovações são normalmente radicais, recebendo inovações incrementais para sustentar o crescimento a partir do atendimento das necessidades e oportunidades do mercado.

O desenvolvimento das inovações e dos relacionamentos acontece por um processo que, no caso da Nexxera, foi identificado como de acordo com os modelos interativo e integrado propostos por Rothwell (1993). O resultado da inovação se reflete pela aceitação pelo mercado refletida no market share e/ou no crescimento do faturamento. Para sustentar sua vantagem competitiva a EBT depende do acesso ao recursos VRIN baseados em conhecimento. No caso da Nexxera: equipe executiva, imagem de marca, domínio de tecnologia, recursos humanos, par- cerias, capacidade de gestão de ativos e acesso ao mercado.

Embora o caminho apresentado na Figura I, e que foi utilizado na avaliação do estudo de caso da Nexxera, tenha se mostrado adequado para identificá-la como uma EBT inovadora, o fato de termos utilizado um estudo de caso é a principal limitação para sugerir que possa ser utilizado genericamente para outras EBT. Sendo assim, sugere-se a realização de trabalhos futuros que possam verificar se o caminho proposto pode ser adequado na análise de outras EBT.

\section{Referências}

ANPROTEC - Associação Nacional de Entidades Promotoras de Empreendimentos de Tecnologias Avançadas. As incubadoras de empresas no Brasil - Panorama 2003. ANPROTEC, 2003.

BAPTISTA, P. A Inovação nos Produtos, Processos e Organizações. Porto: Sociedade Portuguesa de Inovação, 1999.

BARNEY, J. B. Organizational Culture: Can It Be a Source of Sustained Competitive Advantage? Academy of Management Review, I I (3), p. $656-665$, 1986

BARNEY, J. B. Firm resources and competitive advantage. Journal of Management, 17, p. 99 - 120, 1991.

BARTLETT, C. A.; GHOSHAL, G. Managing Across Borders. The Transnational Solution. Harvard Business School Press, Boston, 1989.

BESANKO, D., DRANOVE, D., SHANLEY, M. Economics of Strategy. New York:Wiley, 2000;

CARLSSON, B. Technological Systems and Economic Performance. In: Handbook of Industrial Innovation. Rothwell, R.; Dodgson, M. (eds). UK: Edward Elgar Publishing Company, p. 13 -24, 1994.

CYERT, R. \& MARCH, J. A behavioral theory of the firm. Englewood Cliffs, NJ: Prentice Hall, 1963.

CHANEY, P., T. DEVINNEY,WINER, R. The Impact of New Product Introductions on the Market Value of Firms. Journal of Business, 64, p. 573-610, 1991.

EUROPEAN COMUNITY. Green Book. EEC, 1996.

DAFT, R. L.A dual-core model of organizational innovation. Aca- 
demy of Management Journal. v. 2 I, p. 193-210, 1978.

DAMANPOUR, F.The adoption of technological, administrative, and ancillary innovations: impact of organizational factors. Journal of Management, Washington, D.C., 1987.

DAMANPOUR, F. Organizational Innovation: A Meta- Analysis of Effects of Determinants and Moderators. Academy of Management Journal, Vol. 34, n. 3, p. 555-590, 1991.

DIERICKX, I.; COOL, K.Asset Stock Accumulation and Sustainability of Competitive Advantage. Management Science, Vol. 35, n. I2, p. I504 - I5 I I, 1989.

DOSI, G. Finance, Innovation and Industrial Change. Journal of Economic Behavior and Organization. v. 13, p. 299-319, 1990.

DUNNING, J. H. Reappraising the eclectic paradigm in an age of alliance capitalism. Journal of International Business Studies. Vol. 26, n. 3, p. 46I-49I, 1995.

ETTLIE J. E.; BRIDGES,W. P.; O'KEEFE, R. D. Organization Strategy and Structural Differences for Radical versus Incremental Innovation. Management Science, Vol. 30, n. 6, p. 682-695, 1984.

FREEMAN, C. The economics of technical change: critical survey. Cambridge Journal of Economics, v. I8, p. 463-5 I4, 1994.

GODOY,A. S. Introdução à pesquisa qualitativa e suas possibilidades. Revista de Administração de Empresas, São Paulo, v. 35, n.2, p. 57-63, mar./abr. 1995a.

. Pesquisa qualitativa: tipos fundamentais. Revista de Administração de Empresas, São Paulo, v. 35, n.3, p. 20-29, mai./jun. 1995b.

GRANT, R. Toward a knowledge-based theory of the firm. Strategic Management Journal, v. I 7, p. I09-122, 1996.

HALL, B. The relationship between firm size and firm growth in the US manufacturing sector. Journal of Industrial Economics, $v$. 35, p. 583-606, 1987.

HENDERSON, R.M. e CLARK, K.Architectural Innovation:The Reconfiguration of Existing Product technologies and the Failure of Established Firms. Administrative Science Quarterly, 35, 1990

KIMBERLY, J. R, EVARISKO, M. J. Organizational innovation: the influence of contextual factors on hospital adoption of technological and administrative innovations. Academy of Manage- ment Journal, v. 24, p.689-7I3, I98I.

KLOMP L.;VAN LEEUWEN, G. Linking innovation and firm performance: a new approach. International Journal of the Economics of Business, Vol. 8, n. 3, p. 343-364, 200 I.

LYNN, Gary S.; AKGUN, Ali E. Innovation Strategies under Uncertainty: A Contingency

Approach for New Product Development.Rolla. Engineering Management Journal, Vol. I0, issue 3, p. I I-I7, Sep 1998.

MACHADO, S.A., et al. MPEs de base tecnológica: conceituação, formas de financiamento e análise de casos brasileiros. São Paulo: Sebrae-SP, 200I. Disponível em: <http://www.empresafamiliar.com.br/EMBATEC.pdf.>. Acesso em: 12 out. 2007.

MARCH, J. Exploration and exploitation in organizational learning. Organization Science, n. 2, p. 7I-87, 1991.

MCGRATH, R. ; et al. Innovation, competitive advantage and rent: a model and test. Management Science, v. 42 (3): p. 389403, 1996.

MCGRATH, R. G., MACMILLAN, I. C. The Entrepreneurial Mindset: Strategies for Continuously Creating Opportunity in an Age of Uncertainty. Cambridge: Harvard Business Scholl Press, 2000.

MICHALISIN, M.D., SMITH, R.D., KLINE, D.M. In search of strategic assets. International Journal of Organizational Analysis, vol. 5, no. 4, pp. 360-387, 1997.

MOTOHASHI, K. Innovation Strategy and Business Performance of Japanese Manufacturing Firms. Economics of Innovation and New Technology, vol. 7, pp. 27 - 52, 1998.

NEXXERA. Disponível: http://www.nexxera.com.br/hist.htm . Acesso em: 6 de set. 2006.

NORD, W.R., TUCKER, S. Implementing routine and radical innovation. MA: Lexington Book, 1987.

PENROSE, E. The Theory of the Growth of the Firm. Oxford: Oxford University Press, 1959.

PETERAF, M.A.The Cornerstones of Competitive Advantage:A Resource-BasedView. Strategic Management Journal, I4, p. 179 191, 1993.

PINHO, M. Relatório Setorial Final. Brasília: FINEP.2006. Disponível 
(http://www.finep.gov.br/PortalDPP/relatorio_setorial_final/relatorio_setorial_final_impressao.asp?lst_setor= I6I) acessado em janeiro de 2007.

QUINN, J. B. Managing innovation: controlled chaos. Harvard Business Review, May - June 1985.

REED, R.; DeFILLIPI, R. J. Casual ambiguity, barriers to imitation, and sustainable competitive advantage. Academy of Management Review, v. I5, n. I, p. 88-102, 1990.

ROTHWELL, R. Systems and integration Networking:Towards the Fifth Generation Innovation Process. In: CHAIRE Hydro Quebec Conference en Gestion de la Techonologie. Quebec: University of Montreal, 1993.

ROTHWELL, Roy. Towards the Fifth-Generation Innovation Process. International Marketing Review. Sussex, MCB University Press. Vol. II, n I, pp. 7-3I, I 994.

RUMELT, R. Towards a strategic theory of the firm. In R. B. Lamb (ed.) Competitive Strategic Management. Upper Sadler River, NJ: Prentice Hall, 1984.

SCHUMPETER, J. The Theory of Economic Development. Cambridge: Harvard University Press, 1934.

SCHUMPETER, J. Capitalism, Socialism and Democracy. London: George Allen and Unwin, 1942.

TÁLAMO, J. R.A inovação tecnológica como ferramenta estratégica. Revista Pesquisa e Tecnologia FEl, São Bernardo do Campo, n. 23, p. 26-33, 2002.

TALLMAN, S.; JENKINS, M. HENRY, N. PINCH, S. Knowledge Clusters and Competitive Advantage. Academy of Management Review, v. 29, n.2, p. 258-27I, 2004.

TEECE, D. Capturing value from knowledge assets: The new economy, markets for know-how, and intangible assets. California Management Review, v. 40 (3): p. 55-79, 1998.

TEECE, D. Strategies for managing knowledge assets: The role of firm structure and industrial context. Long Range Planning, v. 33, p. 35-54, 2000.

TEECE, D.J; JORDE, T.M. Innovation and Cooperation: Implications for Competition and Antitrust, Journal of Economic Perspectives. Volume 4, $\mathrm{N}^{\circ} 3,1990$.

TIDD, J; DRIVER, C. Technological and market competencies and financial performance. In: From Knowledge Management to Strategic Competence: Measuring Technological, Market and Organisational Innovation. London: Imperial College Presspp. 94-I25, 2000.

TIDD, J. Innovation management in context: Environment, organization and performance. International Journal of Management Reviews, 3(3), 200I.

TIDD, J., BESSANT, J. e PAVITT, K. Gestão da Inovação: integração das mudanças tecnológicas, de mercado e organizacionais. Lisboa: Monitor, 200I.

UTTERBACK, J.The Process of Innovation:A Study of the Origination and Development of Ideas for New Scientific Instruments. IEEE Transactions on Engineering Management, v. I 8 (4), p. |24-3|, |97|.

VERGARA, S. C. Projetos e relatórios de pesquisa em administração. São Paulo: Editora Atlas, 1997.

WERNERFELT, B.A Resource-Based View of the Firm. Strategic Management Journal, 5, p. I7I - I80, 1984.

YIN, R. K. Estudo de caso: planejamento e método. Porto Alegre: Bookman, 2005. 